\title{
Terapia Ocupacional e qualidade de vida de pessoas com insuficiência renal crônica em hemodiálise ${ }^{1}$
}

\author{
Fernanda Dellê Madalosso ${ }^{a}$, Milton Carlos Mariotti ${ }^{b}$
}

\begin{abstract}
${ }^{\mathrm{a}}$ Graduada, Curso de Terapia Ocupacional, Departamento de Terapia Ocupacional, Universidade Federal do Paraná - UFPR, Bolsista pelo Programa de Iniciação Científica UFPR/TN 2008/2010, Curitiba, PR, Brasil

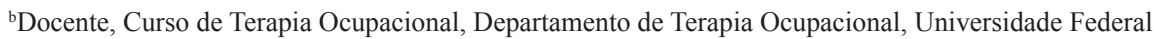
do Paraná - UFPR, Curitiba, PR, Graduado, Terapia Ocupacional, Pontifícia Universidade Católica de Campinas - PUC-Campinas, Campinas, SP, Mestre em Educação e Doutor em Ciências da Saúde, Universidade Federal do Paraná - UFPR, Curitiba, PR, Brasil
\end{abstract}

\begin{abstract}
Resumo: A qualidade de vida dos adultos com insuficiência renal crônica (IRC) em tratamento de hemodiálise apresenta-se comprometida devido a fatores que limitam a funcionalidade e o desempenho de papéis ocupacionais. De acordo com a Sociedade Brasileira de Nefrologia, no Censo de Diálise 2008, existem aproximadamente 41,5 mil usuários em tratamento dialítico, sendo $89,4 \%$ em hemodiálise. A hemodiálise pode ser classificada como uma experiência traumática, que gera dependência e impacto negativo na vida, como a necessidade de dieta alimentar específica, comprometimentos físicos, psicossociais e privação ocupacional. A Terapia Ocupacional visa possibilitar ao indivíduo: promoção do desempenho ocupacional adequado e competente, engajamento e participação em ocupações significativas. Objetivo: Analisar o impacto da intervenção de Terapia Ocupacional na qualidade de vida dos portadores de IRC em hemodiálise. Procedimentos metodológicos: A avaliação foi realizada por meio do questionário de qualidade de vida Kidney Disease Quality of Life - Short Form (KDQOL-SFTM) na sua versão em português, com 28 usuários, em uma clínica de doenças renais do SUS, antes e após a intervenção da Terapia Ocupacional. Resultados: A qualidade de vida, conforme o KDQOL-SFTM, foi avaliada em 19 domínios. Após a intervenção da Terapia Ocupacional, 13 desses domínios obtiveram aumento no escore, sendo que três deles com significância estatística. Conclusões: A qualidade de vida desses usuários apresentou-se comprometida. A Terapia Ocupacional contribuiu para a promoção do aumento na qualidade de vida, portanto é uma profissão competente para atuar com essa população, podendo contribuir para a obtenção de melhora em sua qualidade de vida.
\end{abstract}

Palavras-chave: Terapia Ocupacional, Qualidade de Vida, Insuficiência Renal Crônica.

\section{Occupational Therapy and life quality of patients with chronic kidney disease in hemodialysis}

\begin{abstract}
The quality of life of adults with chronic renal disease (ESRD) under hemodialysis treatment is compromised due to factors that limit the functionality and performance of occupational roles. According to the Brazilian Nephrology Society, the 2008 dialysis census showed that there are approximately 41,500 users of dialysis treatment and that $89.4 \%$ of them are under hemodialysis treatment. Hemodialysis can be classified as a traumatic experience which generates dependency and negative impacts in people's lives, such as dietary restriction, physical and psychosocial commitment, and occupational deprivation. Occupational Therapy proposes the promotion of appropriate and more competent occupational performance, engagement and participation. Objective: To analyze the impact of Occupational Therapy intervention on the quality of life of ESRD patients under hemodialysis treatment. Methodological procedures: Evaluation was performed through the application of the Kidney Disease Quality of Life - Short Form (KDQOL-SF ${ }^{\mathrm{TM}}$ ) questionnaire, in its Portuguese version, to 28 people in a Renal Disease Clinic of
\end{abstract}

Autor para correspondência: Milton Carlos Mariotti, Universidade Federal do Paraná, Av. Lothário Meissner, 632, CEP: 80210-170, Jardim Botânico, Curitiba, PR, Brasil, e-mail: mariotti@ufpr.br 
SUS, before and after Occupational Therapy intervention. Results: Quality of life, according to the KDQOL-SFTM questionnaire, was evaluated in 19 domains. After Occupational Therapy intervention, 13 of these domains showed increased scores: of those, three domains presented statistically significant increases. Conclusions: Initially, the quality of life of the adults evaluated was found to be compromised because of their disease. After the study, it was possible to observe that Occupational Therapy contributed to an increase in the quality of life those people. Therefore, OT is a competent profession to act with the studied population, being able to positively contribute to their quality of life.

Keywords: Occupational Therapy, Quality of Life, Chronic Renal Insufficiency.

\section{Introdução}

As condiçóes de saúde dos usuários adultos com insuficiência renal crônica (IRC) em tratamento de hemodiálise, em geral, interferem no desempenho ocupacional, fato que gera limitações para a realização de atividades de vida diária, produtivas e de lazer, atingindo diretamente sua qualidade de vida. Levando-se em conta que a Terapia Ocupacional atua diretamente com o desempenho ocupacional dos indivíduos visando a qualidade de vida e a funcionalidade, o objetivo deste trabalho foi demonstrar que essa modalidade de intervenção pode ser efetiva com essa clientela.

A hipótese verificada foi a de que há eficácia do atendimento da Terapia Ocupacional na melhora da qualidade de vida dessa população específica. Os usuários que se submetem a hemodiálise apresentam déficits em sua qualidade de vida e podem apresentar melhora significativa através da intervenção de Terapia Ocupacional. Neste estudo, o olhar da Terapia Ocupacional considerou tanto o diagnóstico dos usuários como seus aspectos físicos, mentais e sociais, visando a integralidade do cuidado.

$\mathrm{O}$ instrumento utilizado para avaliar a qualidade de vida foi o questionário Kidney Disease Quality of Life - Short Form (KDQOL-SF ${ }^{\mathrm{TM}}$ ). A partir do questionário específico selecionado foi possível verificar e medir a qualidade de vida da população alvo e comparar os resultados das avaliaçôes realizadas antes e depois das intervençóes da Terapia Ocupacional.

A importância social e científica deste trabalho deve-se à contribuição da Terapia Ocupacional para a melhora da qualidade de vida dessa clientela e ao registro e divulgação do processo, possibilitando o aumento da literatura a respeito da prática da Terapia Ocupacional no campo da qualidade de vida e da população dos usuários com insuficiência renal crônica em tratamento hemodialítico, visto que são escassas as pesquisas e referências bibliográficas sobre esse assunto. Esse fato também poderá auxiliar a subsidiar a criação de políticas públicas na área, com o objetivo de melhorar o atendimento a essa população.

\subsection{Insuficiência renal crônica}

A insuficiência renal crônica (IRC) possui altos índices de morbidade e mortalidade, os quais geram problemas médicos, sociais e econômicos, sendo assim considerada atualmente um problema de saúde pública (CRUZ; PRAXEDES; CRUZ, 2006, p. 248; MARTINS; CESARINO, 2005, p. 671). A IRC é o estágio final de uma lesão progressiva e irreversível da função renal, a qual é responsável por manter a homeostasia do organismo (HIGA et al., 2008, p. 204).

As principais causas da IRC são: a hipertensão arterial, com índice de incidência de 35,8\%, seguida da diabete melitus, com 25,7\% (HIGA et al., 2008, p. 204; SOCIEDADE..., 2008). Em relação aos fatores de risco, os mais importantes a considerar são diabetes e idade avançada (BEZERRA; SANTOS, 2008, p. 687).

Os sintomas e sua progressão variam de acordo com o grau de comprometimento renal, além da presença de comorbidades (KUSUMOTA et al., 2008, p. 153). Esses sintomas geram diversas alteraçôes, tanto fisiológicas como psicossociais, influenciando significativamente os contextos, tanto do usuário como da família, e assim afetando a qualidade de vida deles (HIGA et al., 2008, p. 204; MARTINS; CESARINO, 2005, p. 671).

Os tratamentos são essenciais para a sobrevida, mas possuem um custo bastante elevado, (CRUZ; PRAXEDES; CRUZ, 2006, p. 248), sendo mais de $80 \%$ desse custo subsidiado pelo SUS (SOCIEDADE..., 2008). O tratamento através da hemodiálise será o tipo abordado no presente estudo.

Esse procedimento é realizado no mínimo três vezes por semana, com tempo de duração variando entre $2 \mathrm{~h} 30$ a 4 horas, geralmente em hospitais ou em 
clínicas especializadas (BEZERRA; PIANTINO; MORAIS, 2005, p. 30; CRUZ; PRAXEDES; CRUZ, 2006, p. 278). Pode ser considerado um tratamento sofrido e angustiante (HIGA et al., 2008, p. 206), o qual apresenta durante as sessóes algumas complicaçóes como dores, hipotensão arterial, enjoos e cãibras e, após sua realização, sensação de mal-estar e fraqueza (BEZERRA; PIANTINO; MORAIS, 2005, p. 30; KUSUMOTA et al., 2008, p. 154).

\subsection{Qualidade de vida}

A qualidade de vida tem sido um assunto amplamente estudado, porém não apresenta uma definição universal, pois abrange fatores multidimensionais como transporte, economia, lazer, entre outros, além dos fatores subjetivos (DE CARLO; QUEIROZ; SANTOS, 2007, p. 112-114; MOREIRA et al., 2009, p. 375). De acordo com Minayo, Hartz e Buss (2000), em 1947 a Organizaçáo Mundial de Saúde definiu qualidade de vida como o bem-estar físico, mental e social, independente da ausência de doenças ou enfermidades.

Os instrumentos que avaliam a qualidade de vida relacionada à saúde (QVRS) possibilitam a obtenção de informaçóes específicas das necessidades de saúde, norteando os cuidados e as intervençôes (MOREIRA et al., 2009, p. 375). Existem vários instrumentos diferentes dentro dessa categoria (QVRS), porém, em sua maioria, as especificidades dos acometimentos patológicos não são detectadas, pois são avaliadas por instrumentos categorizados como genéricos, ou seja, não se limitam a uma patologia específica, como o SF-36 e o WHOQOLBref (DUARTE et al., 2003, p. 375).

A qualidade de vida também é determinada pelo enfrentamento da doença, que varia de acordo com as características psicossociais, fatores socioeconômicos, acessibilidade, condiçóes de saúde, esclarecimento patológico e as redes de suporte, como a família, que se destaca apesar de ela variar de acordo com o relacionamento familiar (BOHLKE et al., 2008, p. 255; HIGA et al., 2008, p. 205 e p.206).

\subsubsection{Qualidade de vida dos usuários com insuficiência renal crônica em hemodiálise}

Atualmente, o foco das intervençóes e das pesquisas com essa população está direcionado para o aumento da qualidade de vida desses usuários, ao contrário do foco de antigamente, o qual era inteiramente relacionado à sobrevivência e prolongamento da vida.

O tratamento por hemodiálise não significa necessariamente uma melhor qualidade de vida (MOURA et al., 2006, p. 179; ZÚNIGA et al., 2009, p. 201). A qualidade de vida dos usuários com IRC em hemodiálise está comprometida devido a uma série de fatores que prejudicam a funcionalidade e a vida social, mas principalmente devido ao tempo dispendido nas sessóes de hemodiálise. Esse tratamento pode ser classificado como uma experiência traumática, visto que gera dependência e impactos nas condiçóes de vida, como a limitação nas ocupaçôes e desorganização da rotina, pela restrição alimentar, comprometimentos físicos, como dor, e comprometimentos psicossociais, como bem-estar e satisfação (BEZERRA; SANTOS, 2008, p 687; KUSUMOTA et al., 2008, p. 153; MARTINS CESARINO, 2005, p. 671; TRENTINI et al., 2004, p. 75; VIEIRA et al., 2006, p. 44). As principais queixas dos usuários com IRC em hemodiálise estáo associadas à vitalidade, como falta de energia, ao desânimo e à fadiga, e relacionadas às comorbidades (KUSUMOTA et al., 2008, p. 157 e p. 158). A fadiga é um aspecto a ser considerado pelo alto índice de prevalência e por associar-se a dores nas articulaçóes, à fraqueza muscular (TRENTINI et al., 2004, p. 77), aos sintomas de distúrbios do sono, à depressão e à ansiedade (HIGA et al., 2008, p. 204; MARTINS; CESARINO, 2005, p. 673).

Fatores relativos ao histórico de vida pessoal, frustraçóes e falta de redes de apoio e suporte social e o estresse também podem estar associados ao baixo nível de qualidade de vida (LOPES et al., 2007, p. 508; TRENTINI et al., 2004, p. 75; VIEIRA et al., 2006, p. 44). O estresse pode gerar diversos sentimentos, como de frustração, ociosidade e baixa autoestima, decorrentes da dificuldade de realizar as atividades cotidianas (VIEIRA et al., 2006, p. 44). Pode estar associado ao sentimento de vulnerabilidade em relação ao prognóstico da doença, insegurança em relação aos papéis ocupacionais e preocupação durante o tratamento hemodialítico, pela possibilidade de ocorrerem problemas, como a hipotensão arterial (HIGA et al., 2008, p. 204 e p. 206; KUSUMOTA et al., 2008, p. 154).

A melhora da qualidade de vida evidencia-se através da conquista da autonomia nas atividades inerentes à ocupação de cada indivíduo (MARTINS; CESARINO, 2005, p. 671). Esse fato é percebido nos usuários que apresentam uma atividade produtiva remunerada (BOHLKE et al., 2008, p. 255) e naqueles com esperança de receber um transplante renal (HIGA et al., 2008, p. 206). 


\subsubsection{Kidney Disease and Quality of Life - Short Form (KDQOL-SFTM)}

O instrumento escolhido para avaliar a QVRS de pacientes com IRC em hemodiálise foi o Kidney Disease and Quality of Life - Short Form $\left(\right.$ KDQOL-SF ${ }^{\mathrm{TM}}$ ), por ser amplamente utilizado em diversos países (KUSUMOTA et al., 2008, p. 153; ZÚNIGA et al., 2009, p. 201) e por ser um instrumento específico para avaliar a QVRS dessa clientela.

Esse questionário foi elaborado especificamente para pesquisas com pessoas com insuficiência renal crônica em hemodiálise. Contou com a colaboração de diversos autores, entre eles Ron Hays, e foi escrito originalmente na língua inglesa, em 1995, a partir de entrevistas e revisão de literatura sobre a qualidade de vida desses usuários, náo podendo ser aplicado naqueles que ainda não deram início ao tratamento devido à especificidade das questôes (HAYS et al., 1995, p. 2; DUARTE et al., 2003, p. 379).

O KDQOL-SF ${ }^{\mathrm{TM}}$ é um dos questionários mais completos acerca da qualidade de vida dos portadores de IRC em hemodiálise disponíveis no Brasil e um dos mais utilizados internacionalmente, para essa população, sendo traduzido para diversos idiomas. Esse instrumento permite avaliar dados específicos da rotina dessa população, incluindo todos os tipos de tratamento e transplante (DUARTE et al., 2003, p. 379; DUARTE; CICONELLI; SESSO, 2005, p. 262; KUSUMOTA et al., 2008, p. 153; ZÚNIGA et al., 2009, p. 201).

Esse instrumento apresenta algumas vantagens em relação a outros instrumentos para doenças renais pois já foi aplicado em larga escala, é confiável, validado, permitindo a avaliação em diferentes estágios de tratamento, de acordo com a frequência da aplicação, além de abordar diversos aspectos gerais e específicos, como a sexualidade e a relação com o trabalho (DUARTE et al., 2003, p. 379).

A tradução realizada por Duarte et al. (2003, p. 376) seguiu os padróes estabelecidos pelo KDQOL Working Group, obtidos no próprio website. Sua tradução não acusou dificuldades (DUARTE et al., 2003, p. 377-379; DUARTE; CICONELLI; SESSO, 2005, p. 264).

As questóes de validade, confiabilidade e consistência interna foram observadas e comparadas com outros instrumentos de avaliação já validados para o Brasil, como o Perfil de Saúde de Nottingham (PSN), Escala de Resultados de Karnofsky (ERK) e
Questionário da Doença Renal (KDQ) (DUARTE; CICONELLI; SESSO, 2005, p. 264).

O KDQOL-SF ${ }^{\mathrm{TM}}$ revelou propriedades psicométricas satisfatórias, similares às obtidas em sua versão original. A confiabilidade, assim como em outros países, mostrou-se adequada, através dos índices de consistência interna (DUARTE; CICONELLI; SESSO, 2005, p. 269; MOREIRA et al., 2009, p. 24), apesar da baixa pontuação no item referente ao papel profissional. Esse fato não compromete a aplicaçấo e confiabilidade do instrumento, que pode ser feita por profissionais da saúde (DUARTE; CICONELLI; SESSO, 2005, p. 269; MOREIRA et al., 2009, p. 24, p. 25 e p. 27).

$\mathrm{Na}$ sequência será apresentado o referencial teórico da Terapia Ocupacional e como esta pode intervir no sentido de melhorar a qualidade de vida dessa população.

\subsection{A Terapia Ocupacional}

Para a apresentação do trabalho de Terapia Ocupacional a esses usuários, ressaltando-se que esta foi a primeira experiência de Terapia Ocupacional na instituição, foi importante a delimitação de um referencial teórico para que se pudesse explicitar para eles qual tipo de procedimento seria desenvolvido pelos pesquisadores.

Para o embasamento teórico consideraram-se os estudos referentes à contribuição da Terapia Ocupacional no ambiente hospitalar ou para pessoas dependentes de procedimentos como a hemodiálise para amenizar os impactos e auxiliar na superaçáo das dificuldades encontradas por meio do desenvolvimento de recursos como atividades e ocupaçóes significativas e prazerosas, adaptação ambiental, organização da rotina e auxílio na continuidade da participação social (MORAIS, 2001 apud BEZERRA; PIANTINO; MORAIS, 2005, p. 31; VIEIRA et al., 2006, p. 44).

Assim, delimitou-se o referencial teórico a partir do modelo da ocupação humana e da propriedade terapêutica do engajamento em atividades e ocupaçóes significativas como precursoras de mudanças comportamentais, a fim de manter e promover saúde e funcionalidade (BEZERRA; SANTOS, 2008, p. 688; FERRER; SANTOS, 2007, p. 160; TROMBLY, 2005, p. 256).

Tomou-se ainda como referência a definição de Terapia Ocupacional da Associação de Terapeutas Ocupacionais Finlandeses (WORLD..., 2003, p. 31), por ter sido a única encontrada que engloba o termo qualidade de vida: 
Terapia Ocupacional é uma forma de reabilitação voltada para a habilidade funcional do cliente. Na Terapia Ocupacional o foco é nas habilidades e nos pontos fortes do cliente, em suas limitaçôes, esperanças individuais, demanda do meio ambiente e eliminação de possíveis obstáculos. O objetivo da Terapia Ocupacional é o de promover a qualidade de vida do cliente. O terapeuta ocupacional auxilia-o a buscar e usar sua capacidade máxima nas áreas de cuidados próprios independentes, trabalho e lazer. A meta da Terapia Ocupacional é de que o cliente seja capaz de viver sua vida de acordo com seus valores e tenha uma vida melhor, dentro do possível, independente das limitaçôes causadas por doença, disfunção ou deficiência.

\subsubsection{O modelo da ocupação humana}

A compreensão clara do modelo foi muito importante para o processo de intervenção e destacam-se aqui os principais pontos que apoiaram essa interlocução.

A ocupação, de acordo com esse modelo, é vista como um comportamento humano de uso intencional do tempo, para satisfazer impulsos internos e exigências sociais, a partir da exploração e domínio do ambiente, auxiliando na organização do sistema (FERRARI, 1991, p. 217; KIELHOFNER; BARRET, 2002; KIELHOFNER; BURKE, 1990, p. 56; KIELHOFNER, 1990, p. 117). O comportamento ocupacional é visto, então, como determinante para o ser humano, pois é na ocupação que o indivíduo se habilita e forma seus conceitos e identidade, sendo ela considerada aspecto central da experiência humana (FERRARI, 1991, p. 216; KIELHOFNER; BARRET, 2002; SILVA, 2007, p. 491).

Segundo Kielhofner e Burke (1990, p. 57):

No modelo presente, o sistema representa o homem e a interação do sistema com o ambiente é a ocupação humana. O ambiente é o local físico, social e cultural no qual o sistema opera.

Três subsistemas: volição, hábito e desempenho são hierarquicamente organizados para determinar o comportamento ocupacional, servindo para propósitos diferentes e atuando simultaneamente, interagindo com a ocupação para organizar e regular a ação (KIELHOFNER; BURKE, 1990, p. 57 e p. 58; KIELHOFNER; BARRET, 2002; SILVA, 2007).

As mudanças constantes no sistema preservam a sua integridade e cada sistema aberto possui uma característica constante de mudança resultante de uma ação do sistema. Os fatores que influenciam as mudanças são relativos às demandas do ambiente e às tendências inatas do sistema. A exploração gera habilidades e permite a realização de algo através do prazer, incitando o sistema conforme sua competência e organizando os hábitos em papéis (KIELHOFNER, 1990, p. 114, p. 116 e p. 117). A base do processo de mudanças é o ciclo descrito: estímulo, processamento, reação e novo estímulo, sendo possível gerar ciclos benignos e malévolos.

Assim, no processo de Terapia Ocupacional é possível criar um ambiente propício para desenvolver respostas positivas e incitar a organização dos subsistemas, auxiliando o desenvolvimento de um ciclo benigno, que incentive a exploração de habilidades, para que se reproduza a hierarquia da volição, produzindo novos interesses e papéis ocupacionais de trabalho, lazer e autocuidado em diferentes etapas da vida. Também é possível identificar ciclos malévolos, ou seja, desorganizaçóes ocupacionais, restaurar, manter e realizar ciclos benignos e adaptar as ocupaçóes (FERRARI, 1991, p. 217; KIELHOFNER, 1991, p. 46 e p. 47; KIELHOFNER; BURKE; IGI, 1991, p. 128; SILVA, 2007).

De acordo com Mariotti (2009, p. 20), em sua experiência prática, esse modelo mostrou-se o mais apropriado a ser utilizado na intervenção com essa população específica, pois auxilia a reorganizaçáo do sistema e, principalmente, dos papeis ocupacionais que podem ter sido modificados pela patologia e pelas características do tratamento, possivelmente tendo gerado um ciclo malévolo em relação à adaptação à nova condição, quadro esse que poderá ser revertido com a intervenção da Terapia Ocupacional.

\section{Procedimentos metodológicos}

Trata-se de pesquisa exploratória, de cunho quantitativo, realizada em uma clínica de doenças renais que atende exclusivamente pelo Sistema Único de Saúde - SUS, Brasil, no primeiro semestre de 2010. O número total aproximado de pacientes atendidos era de 240. Sáo seis grupos de usuários, sendo três às segundas, quartas e sextas-feiras e outros três às terças, quintas e sábados. O primeiro horário funciona das $7 \mathrm{~h}$ às $11 \mathrm{~h}$, o segundo, das $11 \mathrm{~h}$ às $15 \mathrm{~h}$ e o terceiro, das $15 \mathrm{~h}$ às $19 \mathrm{~h}$.

Participaram da pesquisa, inicialmente, 33 sujeitos, sendo que três foram transferidos e dois foram transplantados, concluindo-se a pesquisa com 28 sujeitos. 
Foram critérios de inclusão: idade acima de 18 anos e inferior a 60 anos; situaçáo clínica estável; função cognitiva preservada.

Inicialmente, os sujeitos foram avaliados por meio do KDQOL-SF ${ }^{\mathrm{TM}}$, submetidos a intervenção da Terapia Ocupacional, com a média de nove atendimentos, sendo que esses aconteciam semanalmente, durante as sessôes de hemodiálise.

O processo de intervenção seguiu o modelo da ocupação humana, conforme já descrito. Após a aplicação do questionário, buscava-se avaliar a vontade dos usuários em relação às ocupaçóes significativas, assim como suas dificuldades para realizá-las. Uma vez identificadas as ocupaçōes ou atividades de interesse do usuário, essas eram desenvolvidas utilizando atividades construtivas/ manuais, expressivas, jogos, orientação vocacional e profissional.

O ambiente criado durante as sessôes de Terapia Ocupacional possibilitou o exercício das habilidades por meio de atividades adaptadas, de maneira a incitar o sucesso no seu desempenho, visando criar um retorno positivo. Esse processo inicia-se com o respeito aos aspectos do interesse e da vontade, prosseguindo com o desenvolvimento do hábito e finalizando com o desempenho competente.

Após o processo de intervenção da Terapia Ocupacional, os sujeitos foram reavaliados. A partir das avaliaçóes e reavaliaçôes realizadas foram obtidos os escores comparados.

Inicialmente foi realizada análise estatística descritiva, com a análise dos dados sociodemográficos, e, posteriormente, a análise estatística das variáveis. Para cada um dos grupos, a comparaçáo dos resultados da avaliaçáo de qualidade de vida inicial e final deu-se pela aplicação do teste não paramétrico de Wilcoxon, realizado por meio do software SPSS 15.0 for Windows. Valores de $\mathrm{p}<0,05$ indicam significância estatística.

\section{Resultados}

$\mathrm{Na}$ Tabela 1 são apresentados os dados sociodemográficos. Houve predominância de sujeitos casados e com escolaridade até o primeiro grau. A média de idade foi de 50 anos, variando de 28 a 59 anos. O tempo médio de tratamento por hemodiálise apresentado foi de 49,44 meses, ou aproximadamente 4 anos.

$\mathrm{Na}$ Tabela 2 são apresentados os dados relativos aos domínios do instrumento de avaliação KDQOL-SFTM. $\mathrm{Na}$ primeira análise, os domínios mais afetados, ou seja, que apresentaram escores mais baixos, foram:
Tabela 1. Dados sociodemográficos.

\begin{tabular}{lcc}
\hline & $\mathbf{n}$ & $\%$ \\
\hline Sexo & & \\
$\quad$ Feminino & 11 & 39,28 \\
$\quad$ Masculino & 17 & 60,71 \\
Estado Civil & & \\
$\quad$ Casado & 18 & 64,28 \\
$\quad$ Solteiro & 6 & 21,42 \\
$\quad$ Divorciado/ Separado & 4 & 14,28 \\
Escolaridade & & \\
$\quad$ Analfabeto & 4 & 14,28 \\
Até $1^{\circ}$ grau & 20 & 71,42 \\
Até $3^{\circ}$ grau & 4 & 14,28 \\
\hline
\end{tabular}

Fonte: autores.

Situação de trabalho $(19,64)$, Sobrecarga da doença renal $(42,86)$ e Função emocional $(50,00)$. Após a intervenção da Terapia Ocupacional, através de uma média de nove atendimentos, apresentavam-se como domínios mais afetados: Situação de trabalho $(28,57)$, Sobrecarga da doença renal $(47,54)$ e Saúde geral $(52,86)$. Os domínios Situação de trabalho e Sobrecarga da doença renal, embora tenham subido no número dos escores, continuaram sendo os mais afetados.

Os escores mais altos foram atribuídos em ambas as análises aos domínios Suporte social (89,88 na primeira análise e 92,26 na segunda), Função sexual (89,42 na primeira análise e 85,58 na segunda) e Estímulo por parte da equipe de diálise $(85,27$ na primeira análise e 83,04 na segunda).

Das 19 dimensóes do instrumento KDQOL-SF ${ }^{\mathrm{TM}}$, 13 obtiveram aumento nos escores, ou seja, houve melhora em 63,15\% dos aspectos avaliados pelo questionário após a intervenção da Terapia Ocupacional. Um único aspecto permaneceu inalterado, Saúde geral, e apenas em seis aspectos os escores diminuíram: Função sexual, Estímulo por parte da equipe, Satisfação do paciente, Bem-estar emocional, Função social e Energia/fadiga.

Quando submetidos ao tratamento estatístico, os escores mostraram significância estatística em três dos domínios, sendo eles: Lista de sintomas/ problemas, Satisfação do paciente e Dor.

\section{Discussão}

Quanto ao perfil dos usuários, verificou-se serem, em sua maioria, pessoas sem vínculos com atividades produtivas remuneradas, o que contribui para baixa renda. Em relaçáo à escolaridade, a maioria apresentou apenas o ensino básico, indicando 
Tabela 2. Domínios do KDQOL-SFTM.

\begin{tabular}{lccc}
\hline \multicolumn{1}{c}{ Domínios } & Média $1^{\mathbf{a}}$ & Média $\mathbf{2}^{\mathbf{a}}$ & $\mathbf{p}$ \\
\hline 1 Lista de Sintomas/ Problemas & 74,99 & 78,58 & $\mathbf{0 , 0 3 6}$ \\
2 Efeitos da Doença Renal & 67,08 & 68,49 & 0,657 \\
3 Sobrecarga da Doença Renal & 42,86 & 47,54 & 0,238 \\
4 Situação de Trabalho & 19,64 & 28,57 & 0,166 \\
5 Função Cognitiva & 79,52 & 82,38 & 0,369 \\
6 Qualidade da Interação Social & 76,9 & 80,95 & 0,435 \\
7 Função Sexual & 89,42 & 85,58 & 0,658 \\
8 Sono & 75,54 & 76,16 & 0,632 \\
9 Suporte Social & 89,88 & 92,26 & 0,474 \\
10 Estímulo por parte Equipe de diálise & 85,27 & 83,04 & 0,305 \\
11 Satisfação do Paciente & 76,19 & 66,07 & $\mathbf{0 , 0 1 3}$ \\
12 Funcionamento Físico & 55,18 & 61,25 & 0,155 \\
13 Função Física & 50,89 & 56,25 & 0,61 \\
14 Dor & 56,43 & $\mathbf{0 , 0 1 8}$ \\
15 Saúde Geral & 52,86 & 68,57 & 0,779 \\
16 Bem-estar emocional & 69,82 & 52,86 & 0,819 \\
17 Função Emocional & 50 & 68,57 & 0,073 \\
18 Função Social & 79,02 & 66,67 & 0,625 \\
19 Energia/Fadiga & 63,04 & 76,79 & 0,702 \\
\hline
\end{tabular}

Fonte: autores.

baixa escolaridade. Baixa escolaridade e falta de vínculo com atividades produtivas remuneradas geralmente se refletem negativamente nas condições socioeconômicas e, portanto, diretamente na qualidade de vida (BOHLKE et al., 2008, p. 255; HIGA et al., 2008, p. 205 e p. 206).

Estudos que utilizaram como instrumento o KDQOL-SF ${ }^{\mathrm{TM}}$ revelaram menores escores, ou seja, menor qualidade de vida, nos domínios referentes a: Sobrecarga da doença renal, Situação de trabalho e Função sexual (DUARTE; CICONELLI; SESSO, 2005, p. 265; MOREIRA et al., 2009, p. 23). No presente estudo, ratifica-se o achado em relação a Situação de trabalho e Sobrecarga da doença renal. Esse último fato pode estar associado ao perfil pessoal, devido ao baixo índice de vínculo com atividades produtivas e aos sintomas físicos apresentados, como a dor.

O maior escore foi atribuído ao domínio Suporte social, com 92,26 na segunda análise. Esse dado pode estar associado ao fato de os usuários residirem, em sua maioria, com familiares e possuírem companheiro ou serem casados, revelando a importância da rede social para a melhora da qualidade de vida e a inclusão da família no tratamento (BOHLKE et al., 2008, p. 255; HIGA et al., 2008, p. 205 e p. 206).
Após a intervenção da Terapia Ocupacional, os maiores aumentos de escores ocorreram em três domínios: Função emocional $(16,67)$, Dor $(12,14)$ e Situação de trabalho $(8,93)$. Esses fatos podem se interligar ciclicamente. A intervenção pode melhorar a Função emocional, que pode ter relação direta com a intensidade da dor, que consequentemente interfere na autonomia. Como já citado, o aumento da autonomia pode melhorar a qualidade de vida, assim, através de uma atividade produtiva pode-se perceber um aumento também na Função emocional (BOHLKE et al., 2008, p. 255; MARTINS; CESARINO, 2005, p. 671). Esse aumento pode ocorrer com a contribuição do sentimento de empoderamento vivido pelo indivíduo em relação a si próprio, à sua capacidade.

Após a análise estatística, constatou-se diferença significativa $(\mathrm{p}<0,05)$ em três domínios: Lista de sintomas $(\mathrm{p}=0,036)$, Satisfação do paciente ( $\mathrm{p}=0,013)$ e Dor $(\mathrm{p}=0,018)$.

No único estudo encontrado sobre o impacto de um programa de Terapia Ocupacional na qualidade de vida de portadores de IRC em hemodiálise, desenvolvido por um dos autores deste trabalho (MARIOTTI; CARVALHO, 2011), embora utilizando outro instrumento de avaliação, o SF36, constatou-se também mudança significativa no domínio dor $(\mathrm{p}>0,05)$. 
A literatura tem mostrado que a dor crônica é comum para os pacientes com IRC. Tipicamente moderada ou severa, é um problema significativo em mais de 50\% dos pacientes em HD, impactando todos os aspectos da qualidade de vida relacionados à saúde (DAVISON, 2003, 2005). No presente estudo, a dor foi um dos temas centrais, sendo abordada na maioria dos atendimentos por meio de orientaçóes de proteção articular, reorganização da rotina, simplificação de tarefas e conservação de energia.

Em uma avaliação qualitativa, baseada na observação dos participantes, pôde-se perceber de forma geral um aumento significativo na participação em atividades cotidianas e ocupaçôes significativas, assim como maior disponibilidade para a participação social, especialmente no relacionamento com familiares e amigos, influenciando o resultado do aumento dos escores na maioria dos itens abordados pelo KDQOL-SFTM e assim sugerindo a eficácia da Terapia Ocupacional na promoçáo da qualidade de vida.

\section{Conclusões}

O estudo revelou que a qualidade de vida e principalmente a QVRS dos usuários com IRC submetidos a tratamento de hemodiálise apresentam-se comprometidas, devido às alteraçóes físicas e mentais, que levam a alteraçôes nos hábitos de vida, restriçóes na rotina de atividades e ocupaçôes e diminuição da participação social.

De acordo com a revisão bibliográfica realizada sobre o assunto, o estudo do questionário de avaliaçáo Kidney Disease and Quality of Life - Short Form $\left(\mathrm{KDQOL}_{-} \mathrm{SF}^{\mathrm{TM}}\right)$ e da similaridade e correlação dos dados obtidos com as áreas de desempenho ocupacional, concluiu-se que: o KDQOL-SF ${ }^{\mathrm{TM}}$ é um instrumento eficaz para a avaliação da QVRS dos pacientes em hemodiálise, pois auxilia na identificação de déficits, possibilitando, no caso da Terapia Ocupacional, estabelecer os objetivos de maneira mais apropriada, uma vez que há alteraçóes em todas as áreas de desempenho abordadas pela Terapia Ocupacional.

Este estudo corrobora o resultado de estudos anteriores, como se pode verificar através do aumento dos escores na maioria dos domínios do questionário KDQOL-SF ${ }^{\text {TM }}$, com significância estatística em três dos domínios.

Apesar de escassa, especialmente no Brasil, a literatura analisada e a pesquisa de campo demonstraram que o terapeuta ocupacional é um profissional qualificado e indicado para atuar com essa população, promovendo um melhor desempenho ocupacional através de atividades e ocupações significativas e da organização da rotina, assim como do estímulo à participação social. Dessa forma, o processo de Terapia Ocupacional proporciona aumento na qualidade de vida desses pacientes. Entretanto, embora esse seja um potencial campo de atuação do terapeuta ocupacional, sáo raras as referências desse profissional atuando em equipes terapêuticas de atendimento a pacientes em hemodiálise.

Verificou-se que houve efetividade da intervenção da Terapia Ocupacional na qualidade de vida dos usuários atendidos, constatando-se, ainda, a necessidade do desenvolvimento de mais estudos sobre o assunto, para aprofundamento e descriçáo do processo de intervenção, com sua fundamentação teórica para a prática e o estabelecimento de políticas públicas, incluindo-se o terapeuta ocupacional na equipe de atendimento aos usuários em hemodiálise.

\section{Referências}

BEZERRA, K. V.; SANTOS, J. L. F. O cotidiano de pessoas com insuficiência renal crônica em tratamento hemodialítico. Revista Latino-Americana de Enfermagem, Ribeirão Preto, v. 16, n. 4, p. 686-691, 2008.

BEZERRA, K. V.; PIANTINO, D. S.; MORAIS, L. V. Relato de Experiência: Grupo de Terapia Ocupacional Durante Hemodiálise. Revista CETO, São Paulo, n. 9, p. 29-35, 2005. PMid:18833449. http://dx.doi.org/10.1590/ S0104-11692008000400006

BOHLKE, M. et al. Predictors of quality of life among patients on dialysis in southern Brazil. São Paulo Medical Journal, São Paulo, v. 126, n. 5, p. 252-256, set. 2008.

CRUZ, J.; PRAXEDES, J. N.; CRUZ, H. M. M. Nefrologia. 2. ed. São Paulo: Sarvier, 2006.

DAVISON, S. N. Pain in hemodialysis patients: prevalence, cause, severity, and management. American Journal of Kidney Diseases, New York, v. 42, n. 6, p. 1239-47, 2003. PMid:14655196. http://dx.doi.org/10.1053/j. ajkd.2003.08.025

DAVISON, S. N. Chronic Pain in End-Stage Renal Disease. Advances in Chronic Kidney Disease, Philadelphia, v. 12, n. 3, p. 326-334, 2005. http://dx.doi.org/10.1016/j. ackd.2005.03.008

DE CARLO, M. M. R. P.; QUEIROZ, M. E. G.; SANTOS, W. A Terapia Ocupacional em Dor e Cuidados Paliativos - Princípios, Modelos de Intervenção e Perspectivas. In: DE CARLO, M. M. R. P.; QUEIROZ, M. E. G. Dor e Cuidados Paliativos: Terapia Ocupacional e Interdisciplinariedade. São Paulo: Roca, 2007. cap. 5, p. 108-125.

DUARTE, P. S. et al. Tradução e adaptação cultural do instrumento de avaliação de qualidade de vida para pacientes renais crônicos (KDQOL-SFTM). Revista 
da Associação Médica Brasileira, São Paulo, v. 49, n. 4, p. 375-381, 2003.

DUARTE, P. S.; CICONELLI, R. M.; SESSO, R. Cultural adaptation and validation of the "Kidney Disease and Quality of Life - Short Form (KDQOL-SF ${ }^{\mathrm{TM}} 1.3$ )" in Brazil. Brazilian Journal of Medical and Biological Research, Ribeirão Preto, v. 38, n. 2, p. 38-2, Feb 2005.

FERRARI, M. A. C. Kielhofner e o Modelo da Ocupação Humana. Revista de Terapia Ocupacional da USP, São Paulo, v. 2, n. 4, p. 216-219, 1991.

FERRER, A. L.; SANTOS, W. A. Terapia Ocupacional na Atenção a Pacientes com Dor Oncológica em Cuidados Paliativos. In: DE CARLO, M. M. R. P; QUEIROZ, M. E. G. Dor e Cuidados Paliativos: Terapia Ocupacional e Interdisciplinariedade. São Paulo: Roca, 2007. cap. 7, p. 147-166.

HAYS, R. D. et al. Kidney Disease and Quality of Life Short-Form (KDQOL-SF ${ }^{\mathrm{TM}}$ ), version 1.3: A Manual for Using and Scoring. Santa Mônica: RAND, 1995. Disponível em: <http://www.gim.med.ucla.edu/kdqol/>. Acesso em: 05 set. 2009.

HIGA, K. et al. Qualidade de vida de pacientes portadores de insuficiência renal crônica em tratamento de hemodiálise. Acta Paulista Enfermagem, São Paulo, v. 21, p. 203-206, 2008. Número especial. http://dx.doi. org/10.1590/S0103-21002008000500012

KIELHOFNER, G.; BURKE, J. P. Modelo de Ocupação Humana: parte I. Tradução de Ferrari, M. A. C. Revista de Terapia Ocupacional da USP, São Paulo, v. 1, n. 1, p. 55-67, 1990.

KIELHOFNER, G. Modelo de Ocupação Humana: parte II. Tradução de Ferrari, M. A. C. Revista de Terapia Ocupacional da USP, São Paulo, v. 1, n. 2, p. 114-123, 1990.

KIELHOFNER, G. Modelo de Ocupação Humana: parte III. Tradução de Ferrari, M. A. C. Revista de Terapia Ocupacional da USP, São Paulo, v. 2, n. 1, p. 39-49, 1991.

KIELHOFNER, G.; BURKE, J. P.; IGI, C. H. Um Modelo de Ocupação Humana - parte 4 Avaliação e Intervenção (modelo de tratamento, teoria). Traduçáo de Ferrari, M. A. C. Revista de Terapia Ocupacional da USP, São Paulo, v. 2, n. 2-3, p. 127-144, 1991.

KIELHOFNER, G.; BARRET, L. O Modelo da Ocupação Humana. In: WILLARD, H. S.; SPACKMAN, C. S. Terapia Ocupacional. 9. ed. Rio de Janeiro: Guanabara Koogan, 2002. p. 490-492.

KUSUMOTA, L. et al. Adultos e idosos em hemodiálise: avaliaçáo da qualidade de vida relacionada à saúde. Acta Paulista de Enfermagem, Sáo Paulo, v. 21, p. 152-159, 2008. Número especial. http://dx.doi.org/10.1590/ S0103-21002008000500003

LOPES, G. B. et al. Comparaçóes de medidas de qualidade de vida entre mulheres e homens em hemodiálise. Revista da Associação Médica Brasileira, São Paulo, v. 53, n. 6, p. 506-9, 2007. http://dx.doi.org/10.1590/ S0104-42302007000600017
MARIOTTI, M. C. Qualidade de Vida na Hemodiálise: Impacto de um Programa de Terapia Ocupacional. 2009. 80 f. Tese (Doutorado em Medicina Interna e Ciências da Saúde)-Universidade Federal do Paraná, Curitiba, 2009.

MARIOTTI, M. C.; CARVALHO, J. G. R. Improving quality of life in hemodialysis: impact of an occupational therapy program. Scandinavian Journal of Occupational Therapy, London, v. 18, n. 3, p. 172-179, 2011. PMid:20560804. http://dx.doi.org/10.3109/11038128 .2010 .488271

MARTINS, M. R. I.; CESARINO, C. B. Qualidade de vida em pessoas com doença renal crônica em tratamento hemodialítico. Revista Latino Americana de Enfermagem, Ribeirão Preto, v. 13, n. 5, p. 670-676, set./out. 2005.

MINAYO, M. C. S.; HARTZ, Z. M. A.; BUSS, P. M. Qualidade de vida e saúde: um debate necessário. Ciência e Saúde Coletiva, Rio de Janeiro, v. 5, n. 1, p. 7-18, 2000. http://dx.doi.org/10.1590/S1413-81232000000100002 MOREIRA, C. A. et al. Avaliação da propriedade psicométricas básicas para a versão em português do KDQOL-SFTM. Revista da Associação Médica Brasileira, São Paulo, v. 55, n. 1, p.22-28, 2009. http://dx.doi. org/10.1590/S0104-42302009000100010

MOURA, J. A. J. et al. Prevalência de transtornos psiquiátricos em pacientes em hemodiálise no estado da Bahia. Jornal Brasileiro de Psiquiatria, Rio de Janeiro, v. 55, n. 3, p. 178-183, 2006.

SILVA, S. N. P. Análise da Atividade. In: CAVALCANTI, A.; GALVÃO, C. Terapia Ocupacional: Fundamentação e Prática. Rio de Janeiro: Guanabara Koogan, 2007. cap. 12, p. 110-124.

SOCIEDADE BRASILEIRA DE NEFROLOGIA - SBN. Censo de Diálise 2008: Curitiba. São Paulo: SBN, 2008. Disponível em: <http://www.sbn.org.br/index.php>. Acesso em: 15 jun. 2009.

TRENTINI, M. et al. Qualidade de vida de pessoas dependentes de hemodiálise considerando alguns aspectos físicos, sociais e emocionais. Revista Texto e Contextos de Enfermagem, Florianópolis, v. 13, n. 1, p. 74-82, jan./ mar. 2004.

TROMBLY, C. A. Ocupação. In: TROMBLY, C. A.; RADOMSKI, M. V. Terapia Ocupacional para Disfunção Física. 5. ed. São Paulo: Santos, 2005. cap. 11. p. 255-281.

VIEIRA, M. C. et al. Intervenção da terapia ocupacional junto a pacientes com doença renal crônica em tratamento hemodialítico: uma revisão. Revista do Hospital Universitário/UFMA, São Luís, v. 7, n. 1, p. 43-45, jan./ abr. 2006 .

WORLD FEDERATION OF OCCUPATIONAL THERAPISTS - WFOT. Definiçóes de Terapia Ocupacional. São Paulo: Salesiano de Lins, 2003.

ZÚNIGA, C. S. M. et al. Evaluación de la calidad de vida en pacientes en hemodiálisis crónica mediante el cuestionario "Kidney Disease Quality of Life (KDQOL-36)”. Revista Médica de Chile, Santiago, v. 137, n. 2, p. 200-207, fev. 2009. http://dx.doi.org/10.4067/ S0034-98872009000200003 


\section{Contribuição dos Autores}

Fernanda Dellê Madalosso: Participou da elaboração do projeto de pesquisa, da coleta de dados, da intervenção e da redação do texto.

Milton Carlos Mariotti: Responsável pela supervisão e coordenação da elaboração do projeto, desenvolvimento da pesquisa, análise estatística e revisão do texto.

\section{Notas}

${ }^{1}$ Trabalho de pesquisa desenvolvido por meio do programa de Iniciação Científica do Departamento de Terapia Ocupacional da Universidade Federal do Paraná - UFPR, no período 2008-2010. Aspectos éticos: O projeto de pesquisa foi aprovado pelo Comitê Setorial de Ética em Pesquisas em Seres Humanos do Setor de Ciências da Saúde da UFPR e os participantes assinaram o Termo de Consentimento Livre e Esclarecido. Resultados parciais foram apresentados no $18^{\circ}$ Evento de Iniciação Científica - EVINCI, da UFPR. 